\title{
Perforated Meckel diverticulum
}

\author{
Brian T. Kloss • Claire E. Broton • Anne Marie Sullivan
}

Received: 6 May 2010 /Accepted: 21 June 2010 /Published online: 20 August 2010

(C) Springer-Verlag London Ltd 2010

\begin{abstract}
Perforation of a Meckel diverticulum (MD) is a rare complication that can often mimic appendicitis. This case report identifies a child who presented to our Emergency Department (ED) with right lower quadrant abdominal pain, free fluid and air in the abdomen and pelvis, and inflammatory changes visualized on Ultrasonography (US) and computer tomography $(\mathrm{CT})$ scan. In our patient, ruptured appendicitis was suspected, and the diagnosis of ruptured MD was ultimately made by laparoscopy. This case demonstrates that a healthy degree of suspicion for complicated MD should be present when dealing with a questionable diagnosis of appendicitis, particularly in the pediatric population.
\end{abstract}

Keywords Meckel diverticulum .

Meckel diverticulum complications .

Perforated Meckel diverticulum, appendicitis mimic

\section{Case}

A previously healthy 12-year-old male presented to our emergency department as a transfer from an outside facility for a suspected ruptured appendicitis. He initially presented to the outside facility complaining of a sudden onset of stabbing peri-umbilical pain, $10 / 10$ in severity, with a

B. T. Kloss $(\bowtie) \cdot$ C. E. Broton · A. M. Sullivan

Department of Emergency Medicine, Upstate Medical University, 550 East Genesee Street,

Syracuse, NY 13202, USA

e-mail: klossb@upstate.edu

C. E. Broton

e-mail: brotonc@upstate.edu

A. M. Sullivan

e-mail: adena@upstate.edu sudden onset. Up until the onset of the pain he had been in good health, without fever or chills, and had been having normal bowel movements. He denied any associated nausea or vomiting, preceding viral illnesses, diarrhea or bloody stools. Prior to arrival in our department he had been started on broad-spectrum IV antibiotics, and his pain was adequately controlled with IV narcotics (Figs. 1, 2, 3, 4).

On physical exam, the child was resting comfortably, in a seated position. He reported that lying supine exacerbated his pain. His vital signs were as follows: BP $100 / 70$, HR 78, RR 16 and temperature of $36.9^{\circ} \mathrm{C}$. Examination of the abdomen showed some rigidity, voluntary guarding and mild tenderness to palpation in the peri-umbilical area and right lower quadrant. Bowel sounds were present but diminished throughout. The remainder of his physical exam was unremarkable and within normal limits for his age.

Laboratory data were as follows: sodium 138 , potassium 3.7, chloride 99, $\mathrm{CO}_{2} 28, \mathrm{BUN} 9$, creatinine 0.6 and glucose 83 . The complete blood cell count was as follows: white blood cell count 10.8, 63\% neutrophils, hemoglobin 14 , hematocrit 40.5 and platelets 294 . On urinalysis the patient had a trace amount of blood.

An ultrasound from the outside facility showed free fluid in the abdomen and pelvis with an absence of an appendix. The consulting surgical team opted to repeat the abdominal ultrasound study at our facility, which again showed similar results. A CT scan of the abdomen and pelvis was obtained, which demonstrated free air and a moderate amount of free fluid in the pelvis tracking up the gutters. A $1.8 \times 1.0-\mathrm{cm}$ enhancing collection with surrounding inflammatory changes was visualized in the patient's midline.

The patient underwent a diagnostic laparoscopy, which was ultimately converted to an open Meckel diverticulectomy. Under laparoscopy the cecum was located and found 


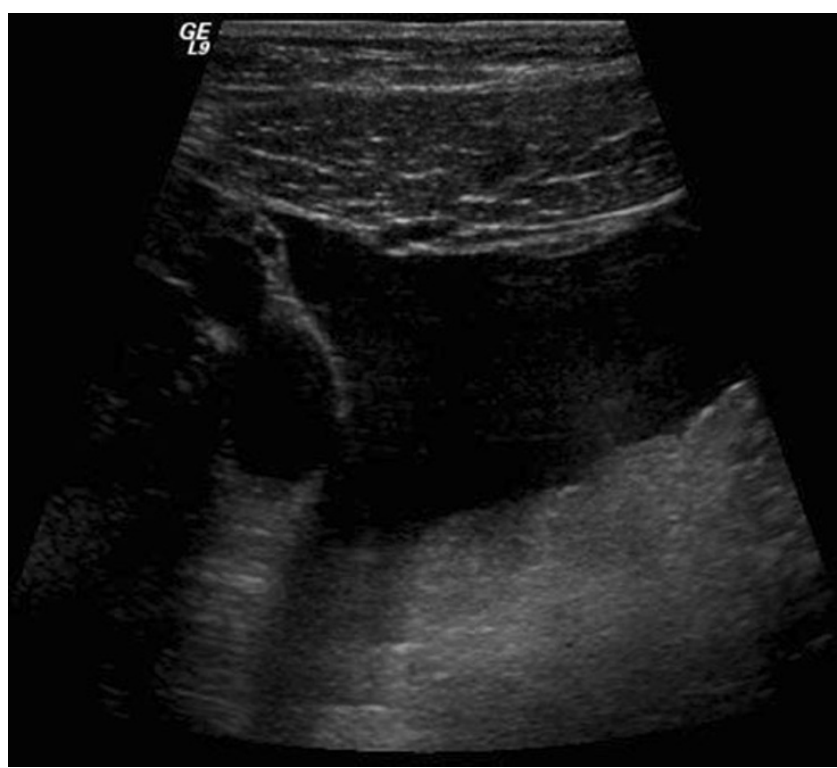

Fig. 1 Ultrasound demonstrates an anechoic area in the right lower quadrant indicating a large amount of free fluid

to be stuck to the side wall of the pelvis. After some dissection, the appendix was identified tacked down in a retrocecal position. It was intact and without any evidence of inflammation. Next the surgeon identified the terminal ileum, which he traced in a retrograde manner to discover a severely inflamed and perforated Meckel diverticulum. At this point in time the surgery was converted to an open laparotomy, and the Meckel diverticulum and appendix were successfully removed and sent to pathology.

After his surgery, the child had a very uneventful hospital course and was ultimately transitioned from IV antibiotics and analgesics to orals and was discharged from the hospital on postoperative day 3 .

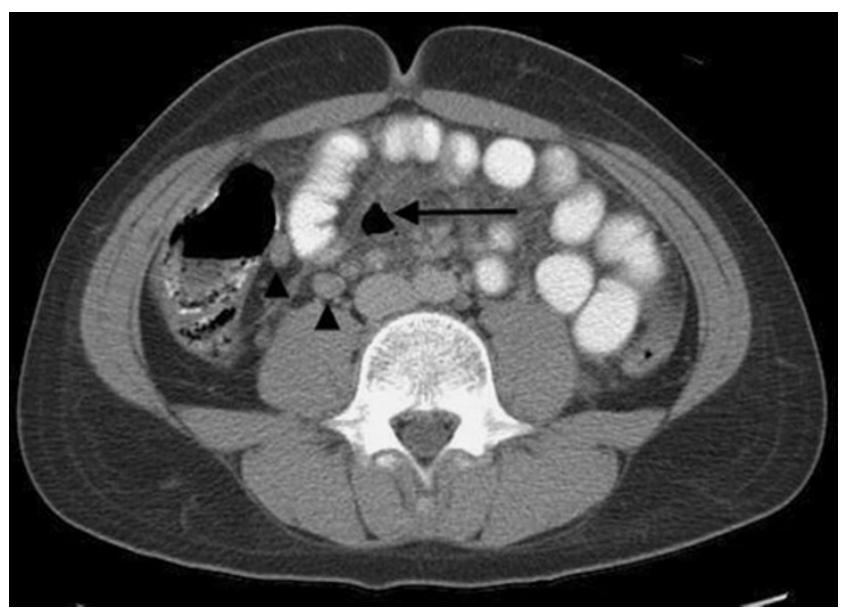

Fig. 2 Enlarged nodes (arrowheads) are seen in the right lower quadrant. A pocket of free air is in the midabdomen (arrow) with surrounding mesenteric inflammation

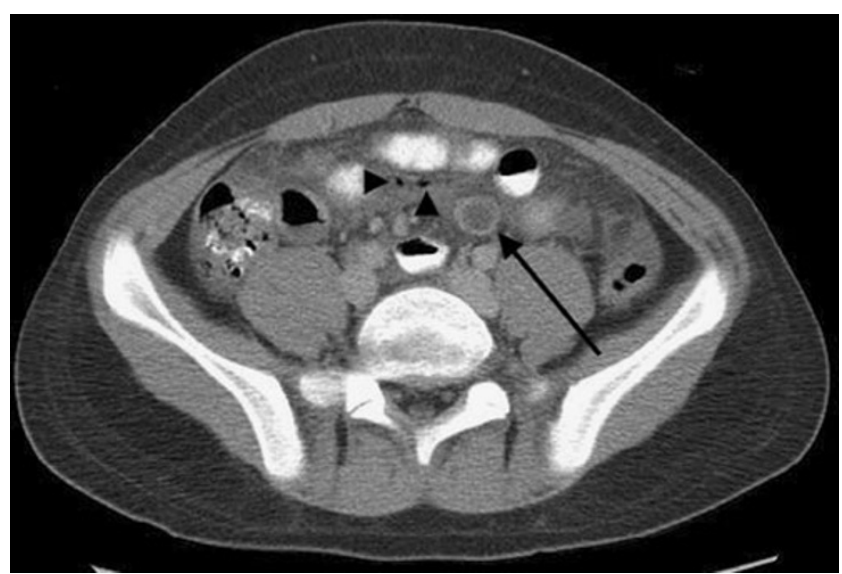

Fig. 3 A fluid-filled, round structure with mural enhancement is indicated by the arrow. Two small foci of extraluminal free air (arrowheads) are consistent with perforation

\section{Discussion}

Meckel's diverticulum (MD) occurs when the omphalomesenteric duct fails to obliterate completely during fetal life. It is the most common congenital abnormality of the gastrointestinal tract, classically thought to represent about $2 \%$ of the population. Despite the fact that this condition is relatively common, only about $4-16 \%$ of cases will lead to complications [1], which include hemorrhage, intussusception, inflammation and, occasionally, perforation, which occurred in our patient. Complications are much more common in males, and the incidence of complications

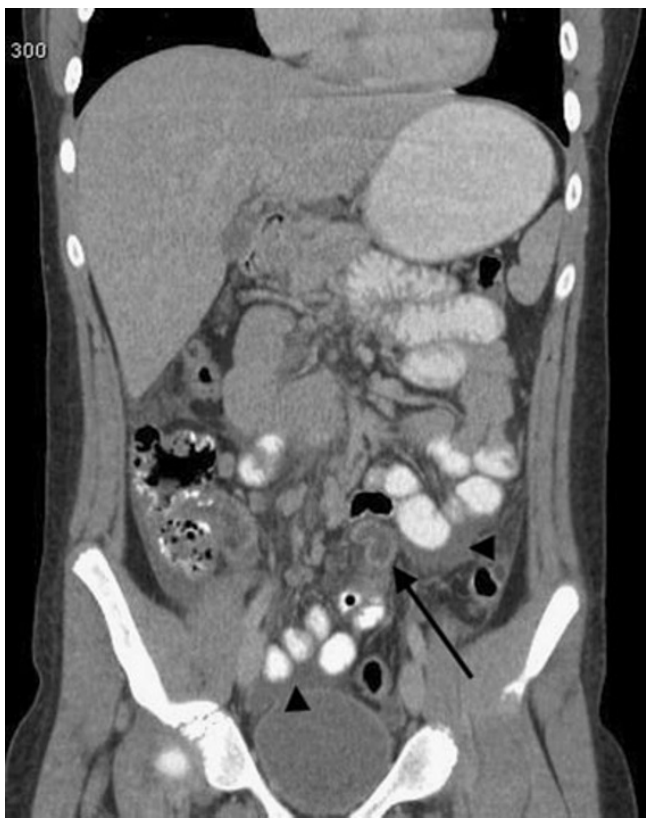

Fig. 4 Coronal imaging demonstrates a blind-ending, tubular structure (arrow) that is fluid filled and demonstrates mural enhancement. Arrowheads indicate free fluid surrounding opacified bowel loops, confirming ultrasound findings 
decreases with age, with the majority occurring in the pediatric population.

The diagnosis of complicated MD presents a number of challenges because of its various presentations. The most common presentation in the pediatric age group is painless rectal bleeding. In a retrospective study of 71 pediatric patients with diagnosed MD, 55.5\% of the patients initially presented with rectal bleeding [2]; however, this number varies among studies $[4,6,8]$. In a study of 776 patients, Kusumoto et al. found that an accurate preoperative diagnosis was made only in $11 \%$ of patients presenting with symptoms other than bleeding, compared to $88 \%$ in bleeding patients [3]. Many of the other presenting symptoms, such as abdominal pain and nausea, are nonspecific and may mimic appendicitis. In one study, $11 \%$ of children with complicated MDs were initially diagnosed with appendicitis [4]. Ueberrueck et al. found that 5 MDs were found incidentally in a series of 311 appendectomies performed at one institution over 3 years [5].

Radiological diagnosis of MD can be difficult, particularly when the diagnosis is not initially suspected. Ultrasound is often used in the setting of non-specific abdominal pain, as it was in our patient; however, it is of limited value for diagnosing MD except in the case of intussusception [6]. A group of ten patients with Meckel's diverticulitis who underwent ultrasound [6] were initially misdiagnosed with appendicitis [7]. CT scan of uncomplicated MD generally resembles a normal loop of the bowel. In the case of diverculitis and perforation, inflammatory changes and extraluminal air may be present [7], but a high degree of suspicion for MD must be present, as this can resemble other common conditions. The technetium- $99 \mathrm{~m}$ pertechnetate scan, or Meckel's scan, is generally regarded as the most accurate, non-invasive diagnostic technique. However, false-negative rates are higher in patients without bleeding [1], and ectopic gastric mucosa must be present in the MD for a positive result.
In our patient, ruptured appendicitis was suspected on CT and ultrasound because of the inflammatory changes, free air and the fact that the appendix could not be visualized. Additionally, the patient had no rectal bleeding. The diagnosis of ruptured MD was ultimately made by laparoscopy. This case demonstrates that a healthy degree of suspicion for complicated MD should be present when dealing with a questionable diagnosis of appendicitis, particularly in the pediatric population.

Acknowledgments This case report did not receive any financial support, and the authors have no conflicts of interest to report. Research was performed at the SUNY Upstate Medical Center Department of Emergency Medicine.

Conflicts of interest None.

\section{References}

1. Sager J, Kumar V, Shah DK (2006) Meckel's diverticulum: a systemic review. J R Soc Med 99:501-505

2. Menezes M, Tareen F, Saeed A, Khan N, Puri P (2008) Symptomatic Meckel's diverticulum in children: a 16-year review. Pediatr Surg Int 24:575-577

3. Kusumoto H, Yoshida M, Takahashi I, Anai H, Maehara Y, Sugimachi K (1992) Complications and diagnosis of Meckel's diverticulum in 776 patients. Am J Surg 164:382-383

4. Sai Prasad TR, Chui CH, Singaporewella FR, Ong CPC, Low Y, Yap TL, Jacobsen AS (2007) Meckel's diverticular complications in children: is laparoscopy the order of the day? Pediatr Surg Int 23:141-147

5. Ueberruek T, Meyer L, Koch A, Hinkel M, Kube R, Gastinger I (2005) The significance of Meckel's diverticulum in appendicitisa retrospective analysis of 233 cases. World J Surg 29:455-458

6. Thurley PD, Halliday KE, Somers JM, Al-Daraji WI, Ilyas M, Broderick NJ (2009) Radiological features of Meckel's diverticulum and its complications. Clin Radiol 64(2):109-118

7. Elsayes KM, Menias CO, Harvin HJ, Francis IR (2007) Imaging manifestations of Meckel's diverticulum. AJR 189:625-629

8. McKay R (2007) High incidence of symptomatic Meckel's diverticulum in patients less than 50 years of age: an indication for resection. Am Surg 73:271-275 